\title{
Ambiente, extensión universitaria y pandemia: reconfiguraciones institucionales en la Universidad Nacional del Litoral
}

\author{
Silvia Leguizamón \\ silmarleg.sı@gmail.com \\ (iD) orcid.org/0000-0002-5184-4911 \\ María Luz Sánchez \\ maluz.bio@gmail.com \\ (DD) orcid.org/0000-0003-4730-5373
}

\author{
Verónica Heinrich \\ veronicah@unl.edu.ar \\ (iD) orcid.org/0000-0002-7805-2682 \\ Universidad Nacional del Litoral, \\ Argentina.
}

\section{Presentación}

La situación de emergencia sanitaria de la pandemia enfoca a las universidades a repensar las trayectorias desde sus funciones sustantivas, docencia, investigación y extensión. Las universidades han tomado un rol proactivo en el territorio en el marco que le brinda su autonomía. En este sentido, el Consejo Interuniversitario Nacional (CIN) destaca la articulación establecida con los Ministerios de Salud, Educación, Desarrollo Social y Tecnológico, como también con los gobiernos locales, para la puesta en marcha de actividades que evaluaron pertinentes respecto de la situación pandémica mundial por COVID-19.

La Universidad Nacional del Litoral (UNL) enfrenta el desafío ante este panorama. Para ello, realiza un intenso trabajo en cuanto a la sistematización de información, planificación de objetivos estratégicos y discusión permanente para reorientar a los equipos de los programas, proyectos y prácticas de extensión en nuevas líneas de acción que implican recrear formas de articulación con las instituciones y organizaciones sociales.

El vínculo permanente con las instituciones y espacios interinstitucionales en el territorio implicó readecuar objetivos y propuestas de extensión universitaria a partir de la articulación de áreas institucionales, unidades académicas, programas y proyectos de extensión en sus diferentes modalidades: Proyectos de Extensión de Interés Social (PEIS); Acciones en el Territorio (AET), Proyectos de Extensión de Interés Institucional (PEII) y Prácticas de Extensión de Educación Experiencial (PEEE).

Es de destacar que en los proyectos de extensión se identifican categorías según la conformación de los equipos académicos participantes. Estas categorías corresponden a: iniciación, experiencia previa y trayectoria en extensión universitaria. ${ }^{1}$ En este esquema abordaremos el trabajo articulado interáreas de la Secretaría de Extensión Social y Cultural (SESyC) (Áreas Programas y Proyectos; Territorio Sociocultural) con dos PEIS que se destacan con las dos últimas categorías, vinculados a distintas unidades académicas de UNL y a programas de extensión. Compartiremos el modo en el que las dinámicas de acciones sostenidas en el tiempo de ambas propuestas permitieron reconfigurar sus prácticas mediante los

\footnotetext{
1) Según la Resolución CS N¹03/19, la categoría “Iniciación en la Extensión Universitaria" incluye todos aquellos equipos de trabajo recientemente constituidos o sin experiencia previa que quieran presentar proyectos. La categoría "Con experiencia previa en extensión universitaria" comprende a todos aquellos equipos que vienen trabajando con proyectos y/o prácticas de extensión con hasta dos convocatorias en proyectos de extensión bianuales y hasta tres convocatorias en caso de proyectos y prácticas de extensión anuales. "Con trayectoria en extensión universitaria" incluye a todos aquellos equipos con trayectoria y experiencia en la extensión universitaria que vienen trabajando con proyectos y/o prácticas de extensión en más de cuatro convocatorias.
} 
conocimientos construidos con y en el territorio. Para ello tomamos la noción de territorio de Mariana Arzeno, quien sostiene que es necesario

“contemplar que el 'territorio de intervención' es, en muchos casos y al mismo tiempo, el territorio apropiado de las organizaciones sociales con las que la universidad interactúa, por lo que la 'extensión en el territorio' tiene que tener en cuenta de las ideas, representaciones, objetivos, vivencias y disputas de esos actores, aun cuando el objeto de la intervención/extensión no sea el espacio en sí. Incluso, pensar el encuentro de saberes en términos de territorio supone concebirnos también nosotros (académicos) como actores que portamos, transitamos y experimentamos múltiples territorialidades que se hacen presentes al momento de pensar la universidad, la sociedad y el espacio que buscamos construir". (2018, p.10)

Desde esta perspectiva retomamos el diálogo con los socios - que son los actores sociales vinculados a nuestros proyectos- ante las nuevas demandas y problemas socioambientales profundizados a partir de la pandemia por COVID- 19.

\section{Universidad y posición institucional}

La UNL asume su compromiso con el sitio litoral centro de la Argentina con vistas de alcance regional, nacional y mundial al incorporar a cada una de las acciones de las políticas, programas y proyectos del Plan Institucional Estratégico (PIE) la dimensión de la "sostenibilidad", que apunta a la reducción del impacto ambiental. Desde el Plan Institucional Estratégico 100+10:

"plantea la misión y la visión a largo plazo que orientan cada una de las acciones. A partir de ellas, se proyecta con 3 líneas institucionales estratégicas (LIE), 15 objetivos institucionales (OI) y 3 ejes transversales (ET). Entre sus ejes transversales la Sostenibilidad ambiental es uno, junto con Inclusión y equidad y Compromiso social, tiene un papel crucial para nuestra universidad". (UNL, 2020, p. 30)

El escenario de pandemia genera una movilización reflexiva crítica que nos implica en la reconfiguración del accionar planificado y hace necesarias discusiones, debates y replanteos organizacionales en diálogo con la sociedad de la cual la UNL es parte. Así, continuando con la idea del reto del contexto actual, es esencial traer al plano de esta narrativa el sostén que brindaron de las definiciones éticas, políticas e institucionales de la extensión universitaria como una de las funciones sustantivas desde sus "múltiples dimensiones: académico-institucional, pedagógica, social, comunicacional y política” (Menéndez, 2017, p. 16) acompañando procesos territoriales como un actor más para potenciar y orientar un accionar transformador como horizonte. Por ello, adherimos a las palabras de la secretaria de Extensión Social y Cultural de la UNL, María Lucila Reyna:

"son momentos en los que se desnudan, con mayor crudeza, la fragilidad del sistema de salud, las inequidades sociales, las brechas tecnológicas y culturales, la debilidad de la política para liderar en estas circunstancias y, muy especialmente, la intemperie física, económica, jurídica y simbólica a la que están sometidos cientos de miles de argentinos.

(...) 
Respecto de las cuestiones que normalmente trabaja la extensión universitaria a través de sus programas y acciones, nos hace pensar directamente en nuevas estrategias de trabajo, en cómo resignificamos el trabajo comunitario en tiempos de pandemia". (2020, pp. 2-3)

La propuesta de extensión, que en su modelo de actuación está basada en el encuentro con las diferentes instituciones, el diálogo permanente con los actores sociales (organizaciones civiles, gubernamentales, redes interinstitucionales e intersectoriales) y el movimiento constante que se genera a partir de agendas de reuniones programadas, se pone en pausa ante las medidas de aislamiento y distanciamiento social obligatorio decretado. De modo que la extensión universitaria

"que a priori sería la mejor preparada para actuar ante una emergencia por estar habituada a las situaciones problemáticas y contar con el repertorio de acción apropiado para apoyar la búsqueda de soluciones colectivas, ahora está en aprietos porque los formatos que usaba hasta hace semanas no responden a lo particular de este caso". (Cano e Ingold, 2020)

Es a partir del respaldo político institucional y de la comprensión reflexiva del contexto que iniciamos espacios virtuales con equipos de gestión presentes, mediados por dispositivos tecnológicos, para posibilitar encuentros.

Apenas iniciada la pandemia en Argentina, la crisis ambiental se profundizó en la región debido a quemas en islas, apariciones y desprotección de especies nativas, problemáticas y demandas sociales que recrudecieron relacionadas con residuos urbanos, alimentación y nuevo paisaje natural por la bajante extraordinaria del río Paraná y de la laguna Setúbal, lo cual fue tomado como una oportunidad para mantener reuniones con responsables de las áreas Programa y proyectos de extensión, Territorio sociocultural y Comunicación, de modo de identificar capacidades dentro de UNL para contribuir en un abordaje posible. Se avanzó contactando a dos equipos de PEIS dada su vinculación con los temas detectados. El criterio de esa elección se basó en que son equipos que, por su conformación, se corresponden con las categorías de "Con experiencia previa..." y "Con trayectoria..." (ver nota al pie 1), lo cuales durante el tiempo de restricciones siguieron gestando opciones en su recorrido por los temas correspondientes y siguieron en permanente y continua comunicación tanto al interior de los propios equipos como con las redes de instituciones con las que tienen un trayecto compartido. Esto hizo que pudieran generar alternativas en la búsqueda de estrategias para la construcción de nuevos escenarios de aprendizajes conjuntos y plantear readecuaciones sobre sus planificaciones previas.

La crisis y la emergencia develaron cambios importantes en la vida cotidiana de ciudadanos, comunidades e instituciones, un fuerte impacto económico y social a causa de las restricciones a la circulación, la suspensión de las instancias presenciales, el distanciamiento y la utilización de elementos personales sanitarios y acciones pertinentes (tapabocas, barbijos, alcohol, lavado de manos, etc.) son algunas medidas con las que convivimos para contribuir a la prevención. En esta situación, la extensión universitaria procuró reacomodarse para estar a la altura de las circunstancias.

Desde esta mirada, problematizamos y priorizamos los ¿cómo? (dimensión metodológica de la intervención social). Los interrogantes empezaron a emerger ante información a veces 
confusa y en otras ocasiones cambiante producto de la pandemia y sus nuevas disposiciones para la circulación en el territorio, medidas generales de prevención, condiciones para el uso del transporte público, programas educativos para los niveles inicial, primario, secundario y superior, recomendaciones para el desarrollo del calendario académico en universidades, etc. Entonces: ¿cómo hacer extensión desde el distanciamiento social? ¿Qué tipo de trabajo territorial es posible sin movilizar docentes y estudiantes al sitio como veníamos realizando? ¿Qué modos de diálogo de saberes y abordajes participativos generar, mediante pantallas, con los actores parte de los proyectos y prácticas?

Como respuesta a algunos de los interrogantes nos planteamos para nuestras prácticas el protagonismo de la dimensión pedagógica de la extensión, de la creación de oportunidades, que tiene como objetivo formar personas, ciudadanos del mundo, y que se habiliten en diálogo con otros para hacer frente, en el marco de "lo posible", a los desafíos que se plantean, y uno de esos retos es la cuestión ambiental.

Hoy la educación transita por diferentes herramientas metodológicas y pedagógicas que tienen como meta colocar a los actores involucrados como verdaderos protagonistas de su aprendizaje. Estas metodologías sustentan dicho aprendizaje en proyectos, y una herramienta fundamental es el aprendizaje basado en la cooperación, el cual permite la relación entre las instituciones y la Universidad, de las instituciones entre sí. En tanto, los procesos de enseñanza y de aprendizaje fundados en su articulación posibilitan estructurar las actividades de manera individual y colectiva.

Una vez instaladas estas prácticas, se consolida una red de aprendizajes que hacen que las acciones sean fuertes, duraderas y significativas en un contexto donde la totalidad de participantes desarrolla valores, capacidades y habilidades, resultados académicos y motivación.

\section{Encuentros necesarios hacia el bien común}

Lo establecido en la Agenda 2030 sobre Desarrollo Sostenible y el Programa XXI (Organización de las Naciones Unidas - ONU-) orienta las políticas de la UNL para priorizar temáticas en los programas de extensión. Ello implica concertar acciones en consonancia con las demandas relevadas de los actores sociales con los cuales se vincula la SESyC. Desde este lugar se propusieron objetivos para el segundo semestre de 2020 partiendo de: "Promover y generar acciones de carácter sociocultural, sostenibles en términos de desarrollo, con especial énfasis en las condiciones generadas en el contexto actual". A su vez, remitir ese propósito hacia aquellas acciones relacionadas con el ambiente desde el objetivo particular de "Generar estrategias y contenidos accesibles sobre prácticas saludables y apoyos necesarios para las personas e instituciones en el marco de la pandemia por COVID-19".

En este escenario cooperativo, donde las estrategias fueron reconfiguradas, tanto los programas y proyectos de extensión como el trabajo de las distintas áreas de la Secretaría se encontraron en un momento de convergencia y de pertinencia en sus acciones.

Es claro que desde la dimensión pedagógica de la extensión un proyecto se concreta a través de un proceso de intervención social, entendido como un conjunto de acciones planificadas e interrelacionadas que se ponen en movimiento para abordar y resolver temas y/o problemas sociales sentidos. 
De esta manera, pensar desde lo ya construido en un espacio de experiencias y saberes compartidos entre actores sociales con historias diferentes pero con puntos en común posibilitó seguir accionando aun ante un hecho inesperado, como una pandemia, que irrumpió en los modelos pedagógicos de educación para dar lugar a nuevas formas de entramar la relación con el conocimiento y en diálogo con los actores sociales.

En este sentido, las mesas de trabajo interáreas de la SESyC, la identificación de actores sociales territoriales (organizaciones civiles y redes interinstitucionales) y la puesta en valor de dos proyectos de extensión de la convocatoria 2019-2020 resignifican los objetivos propuestos.

Aquí presentaremos los modos de abordaje integral de funciones de gestión de área centrales y docencia en el marco de la extensión universitaria. Son las pistas que encontramos para deconstruir y reconstruir los ¿cómo? Fue un trabajo intenso, y para plasmar su recorrido luego de las intervenciones realizadas volvimos al encuentro de los equipos para entrevistar y valorizar su narrativa colaborativa en este artículo.

Estos proyectos de extensión son: PEIS "Derecho a la alimentación. Su promoción a través de huertas agroecológicas y el compostaje”, bajo la dirección de Mirtha Masi, y "Guardianes del río: educación para el fortalecimiento de la identidad insular", cuya directora es Patricia Mines. Fueron muchas las acciones que se produjeron al interior de los proyectos de extensión en vigencia, el apoyo y asistencia permanente de los programas de extensión en cada unidad académica lo hizo posible, y en especial para retomar el contacto con las instituciones del territorio, ya que a partir de las disposiciones de confinamiento, la presencialidad fue tomando formas diversas, a veces difusas y con marcada desigualdad, atravesadas por las Tecnologías de la Información y la Comunicación (TIC).

En un primer momento, el desafío de la virtualidad fue común a todas las áreas de la Universidad en su conjunto, para luego afrontar la misión de esta nueva modalidad en el territorio. No fue una tarea fácil. Coincidimos con Cano e Ingold (2020):

"Dudamos y oscilamos, por ejemplo, entre la necesidad de que las cosas sigan funcionando del modo más normal posible, conservar una cierta cotidianeidad, mantener a raya el miedo; y el criterio de realidad que nos indica que la crisis es muy profunda, y que más que ralentizar algunos de nuestros movimientos por un tiempo, en varios sentidos necesitamos parar".

Pero ese "parar" fue para pensar y para reanudar el movimiento con otros modos.

\section{Diálogo y acciones sobre Patrimonio natural y cultural en tiempos de pandemia con} el PEIS "Guardianes del río: educación para el fortalecimiento de la identidad insular" Hace más de 10 años que en el territorio de la Costa santafesina el proyecto de extensión entrama acciones conjuntas de la mano de sus socios e instituciones de la zona. A partir de la convergencia de los objetivos del Programa de Extensión Ambiente y Sociedad, los proyectos de extensión en vigencia 2019-2020 y PEEE, sumandos a la participación de proyectos de investigación, cumplen con el objetivo de sostener una red para la pertinencia de las acciones en conjunto. Esta situación fue clara y precisa; el modelo pedagógico auténtico fue el puntapié inicial para establecer el diálogo de saberes y la vinculación con el 
territorio en situaciones reales que venían siendo sostenidas en el tiempo. En el comienzo de un nuevo año, 2020, y renovando compromisos una vez más, la pandemia por COVID-19 interrumpió la dinámica y entonces empezó a crearse una modalidad diferente de la acostumbrada. La directora del proyecto, arquitecta y docente de la Facultad de Arquitectura, Diseño y Urbanismo (FADU-UNL), Patricia Mines, refiere:

"No dejamos de encontrarnos con conflictos ambientales; por un lado, una bajante extraordinaria del río y, por otro lado, la quema muchas veces intencional de pastizales en el área insular; los propios conflictos de los pescadores y sus vedas; el aumento de la ocupación de áreas de seguridad hídrica por parte de pobladores de la zona y de manera privada por emprendimientos con autorización dudosa en un escenario que, en paralelo, nos regalaba eventos naturales extraordinarios, como ciertos avistajes de aves exóticas que nos invitaban al asombro". (Entrevista, mayo de 2021)

Luego vinieron los desafíos: el primero comenzó al tomar contacto con los pobladores de La Boca (distrito Alto Verde), hasta hace un tiempo los socios presenciales del proyecto. Los primeros acercamientos fueron telefónicos y por WhatsApp. Luego fue necesario dividir los equipos del proyecto y proponer la creación de una lista telefónica, especialmente con referentes con capacidad y posibilidad de convocatoria y comunicación, como la vecinal y la Escuela Técnico profesional $N^{\circ} 645$ Jacobo Thompson.

El segundo momento fue desde la articulación con el Programa Economía Social y Solidaria y del área Territorio Sociocultural de la SESyC desarrollado a fines de 2019, a través de la generación de una capacitación en Turismo comunitario junto con los vecinos. Se consideró como oportunidad, por el camino recorrido el año anterior, tomar el desafío de la escritura, junto con actores sociales del territorio, de un proyecto para la convocatoria "Puntos de Cultura" del Ministerio de Cultura de la Nación. Por otra parte, también con una institución de Alto Verde, la Asociación "Construir futuro" se gestó una oportunidad de trabajo para la presentación conjunta a una convocatoria a nivel nacional del Ministerio de Ambiente, denominada "Casa Común". El tema que los convocó fue el patrimonio natural y el modo de habitarlo, ya que esa asociación está situada en un lugar que sufre de desmoronamientos debido a su proximidad a la defensa con riesgo hídrico y está sujeta a un futuro planeamiento aún indefinido. Esa es una línea de acción nueva que se suma al Proyecto de Extensión "Guardianes del río".

Al mismo tiempo, en La Boca, conflictos cotidianos se profundizaron con el aislamiento preventivo, de los cuales el más destacado fue el tema de la basura en la laguna. Para encontrar una posible solución, el equipo del proyecto integró grupos de WhatsApp junto a los pobladores y distintos activistas, llamado "Limpieza de la laguna", y otro, "Educación Ambiental en Alto Verde", donde se participó a partir de la escucha activa de reclamos y situaciones-problema hasta llegar a la acción misma de salidas grupales con la actividad específica de limpieza de playas y espacios públicos comunes junto a la gente, al Gobierno de la provincia y a la Municipalidad de Santa Fe.

Ante este panorama, en diálogo con las áreas de la SESyC, y dada la iniciativa de comenzar a promover un espacio de encuentro para el público general con asiento en el trabajo con instituciones en vinculación con el área de Territorio Social y Cultural, surgió el Taller virtual 
denominado "La Naturaleza empieza en casa". ${ }^{2}$ El planteo de base de esta propuesta fue un abordaje de la temática desde

"un relato desde los aspectos naturales, pero además poder relacionar la idea de naturaleza no solamente con lo natural y lo biológico, sino también esa dimensión cultural, que se viene trabajando desde el Programa Ambiente y Sociedad". (Entrevista a Patricia Mines, mayo de 2021)

El Taller se trató de dos encuentros de una hora y media mediante la plataforma Zoom y con transmisión directa por el canal de YouTube de la SESyC (2020). Implicó el acercamiento a las especies nativas diferenciando los riesgos respecto de aquellas consideradas "peligrosas", como serpientes de la zona, las que ante los incendios en su hábitat natural aparecieron y llegaron a las viviendas, algo nunca antes visto. Por otra parte, considerando la trayectoria del proyecto de extensión, se expusieron orientaciones para comprender y analizar cómo estamos situados en nuestra propia casa, manzana, barrio, ciudad, hasta llegar a dimensiones que integren una visión macro de nuestro planeta y reflexionando que desde nuestro propio lugar es posible contribuir en cuanto al ambiente y al desarrollo sostenible, considerando que hay algunos grises en la cuestión y no solo polos antagónicos.

El trabajo continúa porque la visión del patrimonio natural y cultural de las islas del valle del río Paraná es la idea fuerza de trabajo conjunto del desarrollo sostenible articulado desde el Programa Ambiente y Sociedad, en diálogo con otros dispositivos institucionales (áreas centrales, proyectos, programas, cátedras, unidades académicas) que permiten la integración de funciones (extensión, investigación y docencia). Así, este recorrido también ha permitido el fortalecimiento interno y seguir consolidando acciones con la comunidad de Alto Verde (La Boca) y con la elaboración del PEII "Paisaje de islas: Patrimonio y turismo comunitario como estrategia de desarrollo solidario", ${ }^{3}$ surgido de una convocatoria especial en el contexto de emergencia sanitaria.

\section{Diálogo y acciones sobre soberanía alimentaria y producción agroecológica en tiempos de pandemia con el PEIS "Derecho a la alimentación. Su promoción a través de huertas agroecológicas y el compostaje"}

Desde 2016, y en conjunto con la Municipalidad de Santa Fe, se viene acompañando al Programa de Huertas en la promoción de la siembra familiar. En un inicio, la idea fue alcanzar a todos los barrios de la ciudad, y muchos fueron los pasos para la consolidación del proyecto. Desde la vinculación con la Agroecología a partir de la materia Derecho agrario, dictada por la directora del PEIS Mirtha Masi de la Facultad de Ciencias Jurídicas y Sociales (FCJSUNL), se conformó un equipo de extensión interdisciplinario que dio lugar al abordaje de la soberanía alimentaria mediante el seguimiento y talleres para saber qué pasaba en principio con el destino de las semillas.

2) Coordinados por la Lic. en Biodiversidad Alba Imhof y la Arq. Patricia Mines, integrantes del Programa Ambiente y Sociedad.

3) UNL. Resolución CS N 96/21. 
Esta primera experiencia se enriqueció con la conformación del grupo ya que logró una fusión y una idea de trabajo compartido —cuestiones que no siempre se consiguen—, lo cual fue uno de los valores iniciales del proyecto.

Consolidado el equipo de extensión, surgieron en el territorio nuevas demandas y se focalizó el trabajo con la Asociación Vecinal Juventud del Norte. En palabras de la directora:

"Tuvimos muchísimos voluntarios participando, incluso algunos internacionales, que además lograron involucrarse de manera muy fuerte potenciando al proyecto, además de la incorporación de docentes investigadores y su aporte en las líneas de trabajo sobre derecho a la alimentación, lo que posibilitó trabajar más sobre cuestiones y marcos teóricos para replantear en el territorio". (Entrevista a Mirtha Massi, mayo de 2021)

Este flujo de información fue necesario para nutrir el proyecto de cuestiones académicas y en relación permanente con la asociación civil socia. La misma está estratégicamente ubicada en un terreno propio que posibilitó la instalación de una huerta que, con el correr de las intervenciones, se fue constituyendo en modelo, apta para ser replicada en otros lugares. La presencia en el barrio también consistió en una invitación personalizada a los vecinos desde la UNL y el Instituto de Nacional de Tecnología Agropecuaria (INTA) Santa Fe. Las posteriores capacitaciones y encuentros afines permitieron la vinculación con la organización Casa de Semillas del Litoral, que tiene como finalidad revalorizar las semillas de especies de plantas nativas, semillas libres, ${ }^{4} \mathrm{y}$ el acceso a las semillas a partir del intercambio.

Al pensar la Huerta Agroecológica se generaron producciones de contenidos y piezas comunicacionales que se acercaron a la vecinal. Retomando la voz de la directora del proyecto:

"Este proceso se vio interrumpido cuando nos topamos con el inicio del año 2020, siendo un cimbronazo tan grande en el que nos preguntamos ¿qué hacemos?, y las respuestas eran pensadas desde la virtualidad. Contamos con la ventaja de que en plena pandemia el Programa Prohuerta continuaba acciones en el territorio". (Entrevista a Mirtha Massi, mayo de 2021)

Mediante la comunicación permanente se propuso, a partir de la duplicación de la demanda de semillas, una serie de capacitaciones y la creación y armado de un material específico para docentes de las escuelas, especialmente aquellas ubicadas en zonas más vulnerables de la ciudad. La finalidad principal fue que cada niño y niña descubriera el valor de la semilla y su potencia de vida. La estrategia de pensar en un cuento, un relato corto, derivó en un audiocuento ${ }^{5}$ con ilustraciones de una diseñadora gráfica que se integró a la producción de este material que contenía un mini-kit de semillas. Las escuelas interesadas asistían a capacitaciones por medio distintas plataformas y luego se pactaba la entrega del kit completo. Allí la virtualidad seguía su curso. Esta propuesta fue un acercamiento a aquellos docentes con la idea de adaptar sus secuencias de enseñanza para que los niños y las niñas pudieran conocer el paso a paso de ese contenido.

Al trabajo en equipo del PEIS se sumó la participación de las diferentes áreas de la SESyC, y el teletrabajo fue un desafío al momento de acordar, difundir y ejecutar las acciones colabo-

4) Son aquellas que no se comercializan, que están a disposición de la comunidad. 5) El audiocuento fue diseñado para circular a través del WhatsApp. 
rativamente con otros actores sociales con los cuales el proyecto estuvo dispuesto a ampliar territorio por medio de la capacitación virtual. Por otra parte, se articuló con el área Territorio Sociocultural la entrega de kit de semillas a referentes de instituciones del distrito noroeste que colaboraron en la convocatoria de vecinos participantes del espacio de formación.

\section{Desafíos}

El contexto de la pandemia ineludiblemente generó una suerte de caos que obligó a todos los protagonistas de la comunidad universitaria a transitar un proceso de reflexión y empatía con relación a lo que se venía haciendo, y se pusieron en marcha proyectos nuevos para brindar visibilidad al compromiso social de la Universidad con el entramado del cual es parte.

Dos PEIS y una mesa de diálogo permanente interáreas de la SESyC denotaron en su praxis la contribución a los nuevos problemas socioambientales surgidos durante la emergencia sanitaria.

La apuesta hacia esta novedosa mirada en los modos de entender la educación, para conocer y aprender de manera más reflexiva, cooperativa y solidaria la problemática, pone de manifiesto las estrategias reconfiguradas y la articulación de las distintas áreas de trabajo de la SESyC, programas, proyectos y prácticas de extensión, lo cual contribuyó a profundizar la dimensión pedagógica de la extensión:

"Así, entender la educación, conocer y aprender implica comprometerse en un mismo proceso cambiante, difícil e incierto, donde el conocimiento y el aprendizaje se constituyen en campos también complejos y problemáticos. El conocimiento transcurre dentro de los sistemas de actividad que se desenvuelven social, cultural e históricamente, e involucra a personas que se vinculan de manera múltiple y heterogénea. En el recorrido dado, cómo se aprende, es posible mostrar que se pueden crear nuevos conocimientos interviniendo en la práctica, en la actividad misma; de allí la necesidad de participar en escenarios sociales amplios. En este sentido, se demuestra que puede existir un potencial educativo muy grande al momento de intervenir desde la educación formal en prácticas sociales concretas, donde el conocimiento circula, se transforma, se crea y recrea" (Menéndez 2017, pp 31-32)

Desde esta visión, el fortalecimiento de la extensión universitaria con una mirada crítica y reflexiva hacia el ambiente nos insta a repensar, crear y recrear herramientas metodológicas de planificación para el diseño y construcción de los procesos de intervención social que permitan definir más claramente cada una de las prácticas de extensión, con capacidad de anticipar e identificar problemas o conflictos, de analizar su viabilidad y sostenibilidad, profundizando el involucramiento, el diálogo y la construcción innovadora del conocimiento.

\section{Referencias bibliográficas}

Arzeno, M. (2018). Extensión en el territorio y territorio en la extensión. Aportes a la discusión desde el campo de la Geografía. +E: Revista de Extensión Universitaria, 8(8), 3-11. 10.14409/extension.v8i8. Ene-Jun.7709

Cano, A. e Ingold, M. (2020). La extensión universitaria en tiempos de pandemia: lo que emerge de la emergencia. Redes de Extensión, (7), 38-45. Sección Especial. 
Menéndez, G. (Comp.) (2013). Integración docencia y extensión. Otra forma de aprender y de enseñar. Ediciones UNL.

Menéndez, G. (2017). Resignificación de la extensión a 100 años de la Reforma Universitaria de 1918. +E Revista de Extensión Universitaria, 7(7), 24-37.

ONU Agenda 2030. Desarrollo sostenible ONU. LC/G.2681-P/Rev.3.

Programa Siglo XXI (1992). Conferencia de Naciones Unidas sobre el Medio Ambiente y el Desarrollo (UNCED). Reyna, M. (2020). La extensión universitaria en emergencia(s). Pensar y trabajar con las comunidades en contextos de pandemia desde la Universidad Nacional del Litoral. +E: Revista de Extensión Universitaria, 10(12). https://doi.org/10.14409/extension.v10i12.Ene-Jun.9261

SESyC, UNL (2020). Taller "La naturaleza empieza en casa". Patricia Mines y Alba Imhof [video]. Youtube. https://www.youtube.com/watch?v=xAEhTbycf-I/https://www.youtube.com/watch?v=JLefsfY_Av8

UNL (2020). Plan Institucional Estratégico 100+10 (2020). Resolución CS N³40/20. Expediente REC1047199-20. 